\title{
Prediction of photothermal phase signatures from arbitrary plasmonic nanoparticles and experimental verification
}

\begin{abstract}
Omry Blum and Natan T Shaked
We present a new approach for predicting spatial phase signals originating from photothermally excited metallic nanoparticles of arbitrary shapes and sizes. The heat emitted from such a nanoparticle affects the measured optical phase signal via changes in both the refractive index and thickness of the nanoparticle surroundings. Because these particles can be bio-functionalized to bind certain biological cell components, they can be used for biomedical imaging with molecular specificity, as new nanoscopy labels, and for photothermal therapy. Predicting the ideal nanoparticle parameters requires a model that computes the thermal and phase distributions around the particle, thereby enabling more efficient phase imaging of plasmonic nanoparticles and avoiding trial-and-error experiments while using unsuitable nanoparticles. The proposed nonlinear model is the first to enable the prediction of phase signatures from nanoparticles with arbitrary parameters. The model is based on a finite-volume method for geometry discretization and an implicit backward Euler method for solving the transient inhomogeneous heat equation, followed by calculation of the accumulative phase signal. To validate the model, we compared its results with experimental results obtained for gold nanorods of various concentrations, which we acquired using a custom-built wide-field interferometric phase microscopy system. Light: Science \& Applications (2015) 4, e322; doi:10.1038/lsa.2015.95; published online 28 August 2015
\end{abstract}

Keywords: digital holographic microscopy; interferometric imaging; nanoparticles; phase measurement; plasmonics

\section{INTRODUCTION}

Plasmonic nanoparticles are used in a variety of scientific disciplines because of their unique interactions with electromagnetic fields. Plasmonic nanoparticles are used in photonics to sense or induce changes in certain environments, thereby acting as either nanosensors or nanosources for changes in chemical, thermal, or material properties $^{1}$. In biomedical applications, plasmonic nanoparticles can be used as labels in cells and tissues and are imaged via various effects, including photothermal (PT) imaging, photoacoustic shock-wave imaging, scattering, and polarization imaging ${ }^{2-5}$.

The electromagnetic energy absorbed by a metallic nanoparticle is rapidly transformed into heat through electron-phonon relaxation; thus, nanoparticles in solution act as nanosources of heat, which can be manipulated for imaging or for destructive purposes.

Several numerical models of nanoparticles have been proposed to estimate the transfer of heat from a nanoparticle submerged in a liquid to its surroundings ${ }^{5-8}$. These models can yield information on the processes the nanoparticles undergo on time scales or at resolutions smaller than those at which they can be measured. In addition, these models can predict the thermal distribution in the nanoparticle surroundings; thereby making it possible to explain phenomena observed when heated particles interact with other materials, particularly with biological materials that are prone to thermal damage.
When nanoparticles are conjugated to biological carriers such as antigens, they can attach to the appropriate biological cell receptors; thereby enabling molecularly specific excitation of these nanoparticle/ receptor complexes using light ${ }^{9,10}$. Because of the correlation between the local temperature change in the solution surrounding such a nanoparticle and the phase of the light interacting with the solution, it is possible to image these nanoparticles and detect their locations. Hence, imaging the nanoparticles as heat sources can be accomplished using phase-sensitive techniques such as differential interference contrast microscopy ${ }^{11,12}$, phase-sensitive optical coherence microscopy $^{13,14}$, and wide-field interferometric phase microscopy ${ }^{15}$. In these PT phase-imaging methods, the nanoparticles are stimulated at their peak plasmonic wavelength by time-modulated illumination. The optical excitation of the nanoparticles induces absorption, yielding a temperature rise in the nanoparticle surroundings, which causes phase changes that can be detected optically.

Optimization and enhancement of the nanoparticle phase signal is expected to improve the imaging capabilities of these phase-sensitive techniques. This can be achieved through careful design of the nanoparticle materials, shapes, and sizes, which greatly affect the nanoparticle optical properties and the resulting phase signal. Instead of synthesizing many types of nanoparticles and testing them experimentally, simulation tools for prior evaluation of the expected thermal 
and phase signals can be used. Only after simulative inspection and optimization of the nanoparticles, should the experimental synthesis of the chosen nanoparticles be performed. However, to the best of our knowledge, there is currently no study that has simulated the quantitative optical phase profiles extracted from heated nanoparticles of arbitrary parameters with the purpose of optimizing the nanoparticle shape, size, and the related optical phase-imaging system parameters, and allowing for the optimization of nanoparticles for specific needs, for example, for producing the strongest possible phase signal for phase imaging without inducing a damaging temperature rise, or alternatively, for obtaining highly localized temperature for PT therapy.

This study suggests a computational model that predicts the quantitative phase profiles of photothermally excited plasmonic nanoparticles of arbitrary shapes. The model is based on a finite-volume method for geometry discretization and an implicit backward Euler method for solving the transient inhomogeneous heat equation. The local change in heat is then related to the phase change via changes in both refractive index and thickness. Experimental validation of the model is performed through wide-field phase imaging of gold nanorods using an interferometric microscopy system.

\section{MATERIALS AND METHODS}

\section{Heat model}

Our heat model assumes that small solid particles with a high surfaceto-volume ratio that are situated within a liquid medium tend to experience strong frictional forces, which rapidly minimize their speed relative to the medium. This behavior is relevant for nanoparticles suspended in a fluid, and as such, they remain stationary within the surrounding medium for the duration of the measurement. Heat transfer via convection occurs because of the relative movement of two adjacent materials, from which we deduce that the omission of heat transfer via convection in this model is acceptable. For simplicity, we assume that the nanoparticles do not occlude each other and do not aggregate. Otherwise, both their cross section and their spectral absorption might be altered.

Biological tissue is sensitive to changes in temperature. A temperature rise of more than $5{ }^{\circ} \mathrm{C}$ above homeostasis causes tissue damage over time ${ }^{16}$. Short and powerful laser-induced pulses over a period of a few nanoseconds, at energies as low as $1 \mathrm{~mJ}$, can cause tissue damage due to heat build-up ${ }^{17}$. A temperature rise on the surface of a nanoparticle of above $100{ }^{\circ} \mathrm{C}$ induces water vaporization. During thermal stimulation of nanoparticles suspended in aqueous solution to excessive temperatures, vapor bubbles may form around the nanoparticles, which change the dielectric constant of the surrounding material and the nanoparticle extinction and absorption coefficients ${ }^{18}$. This vapor formation may cause strong cavitation and pressure waves that can damage the surrounding tissue. A heat transfer model of spherical nanoparticles that considers these effects has been discussed previously ${ }^{7}$. Our model, however, avoids these cases and assumes that the temperature of the photothermally stimulated nanoparticles suspended in medium does not exceed $100{ }^{\circ} \mathrm{C}$ and that vapor formation is therefore negligible. Because of this lack of a vapor layer, we assume boundary-free surroundings around the nanoparticles; thus implying uniform temperature and energy flux at the particle-medium boundary.

In addition, we assume that the absorption coefficient and the cross section of the nanoparticle are known for all wavelengths. We can then assume excitation of the particle at its peak absorption wavelength, causing surface plasmon resonance (SPR).
As mentioned above, because we assume that convection heat transfer is negligible at the nanoscale, conduction dominates the thermal distribution problem. When considering many identical heat sources, one can first find the thermal distribution solution for a single source and then use superposition to derive the collective solution. Because the proposed model was designed primarily for the investigation of phase signals originating from photothermally stimulated nanoparticles, we ultimately applied a conversion from the amount of heat to the accumulated phase.

For discretization, we chose the finite-volume method on a grid containing Cartesian cubic voxels of constant volume. This method is particularly accurate for energy conservation equations, such as the heat equation ${ }^{19}$.

Under these conditions, the transient inhomogeneous, internalheat-generation, conduction-only heat equation is:

$$
\rho C_{p} \frac{\partial T}{\partial t}=k \nabla^{2} T+S
$$

where $\rho$ is the density of the material (i.e., water/gold) (in $\mathrm{kg} \times \mathrm{m}^{-3}$ ); $C_{p}$ is the specific heat of the material at constant pressure (in $\mathrm{J} \times$ $\left.\left({ }^{\circ} \mathrm{K} \times \mathrm{kg}\right)^{-1}\right) ; T$ is the temperature of the material (in ${ }^{\circ} \mathrm{K}$ ); $\kappa$ is the thermal conductivity of the material (in $\left.\mathrm{W} \times\left({ }^{\mathrm{K}} \times \mathrm{m}\right)^{-1}\right) ; \nabla^{2}$ is the Laplace operator, which is equal to $\partial^{2} T / \partial x^{2}+\partial^{2} T / \partial y^{2}+\partial^{2} T / \partial z^{2}$ in Cartesian coordinates; and $S$ is the term representing the heat generated due to laser excitation (in $\mathrm{W} \times \mathrm{m}^{-3}$ ). This equation applies within two regions, the nanoparticle region and the medium region. Each region has its own coefficients for material density $\rho$, specific heat $C_{p}$, thermal conductivity $\kappa$, and heat generation $S$. In the nanoparticle region, $S$ is calculated from the energy absorbed by the particle in accordance with its cross section. This parameter is calculated as follows:

$$
S=(I \times C) /(A \times V)
$$

where $I$ is the excitation laser intensity (in W), $A$ is the area of the excitation laser spot $\left(\right.$ in $\left.\mathrm{m}^{2}\right), C$ is the optical cross section of the particle (in $\mathrm{m}^{2}$ ), and $V$ is the particle volume (in $\mathrm{m}^{3}$ ). The optical cross section $C$ of the particle can be calculated using either an analytical method, if possible, such as the Mie theory, or a numerical method, such as the discrete dipole approximation. In the surrounding medium region, $S$ is considered to be zero; thus, we assume that the direct heating of the medium due to the laser illumination is negligible.

Analytical solutions for Equation (1) may be derived, with great effort, only for highly symmetric shapes. As an alternative, numerical methods can be applied to solve this equation for arbitrary shapes, non-uniform media, and time-varying heat-source terms. In general, numerical methods for solving the heat equation can be divided into explicit and implicit methods. In explicit methods, the solution for the next time step is derived straightforwardly from that for the present time step; whereas in implicit methods, the solution for the next time step is derived by solving a system of equations constructed from the present time step, connecting all elements on a grid to the next time step solution. In our model, we use an implicit backward Euler method to solve the heat equation ${ }^{19}$. Because we use time-modulated illumination to stimulate the nanoparticles, we have an oscillating timedependent heat-source term $S$. The derivation of the temperature is discretized in space using the three-point, second-order central difference approximation, which introduces a truncation error of $O(h)$, where $h$ is the characteristic size of a voxel ${ }^{20}$. In this case, for each voxel located at $r=(x, y, z)$, the product of $\kappa$ with the Laplace operator 
in Equation (1) can be rewritten as a linear combination of its neighboring voxels, as follows:

$$
\begin{aligned}
k \nabla_{r}^{2} T= & \frac{1}{h^{2}}\left[k_{x+0.5, y, z}\left(T_{x+1, y, z}-T_{x, y, z}\right)+k_{x-0.5, y, z}\left(T_{x-1, y, z}-T_{x, y, z}\right)+\right. \\
& k_{x, y+0.5, z}\left(T_{x, y+1, z}-T_{x, y, z}\right)+k_{x, y-0.5, z}\left(T_{x, y-1, z}-T_{x, y, z}\right)+ \\
& \left.k_{x, y, z+0.5}\left(T_{x, y, z+1}-T_{x, y, z}\right)+k_{x, y, z-0.5}\left(T_{x, y, z-1}-T_{x, y, z}\right)\right]
\end{aligned}
$$

The thermal conductivity may change with each location on the grid, as it depends upon the materials being modeled. As such, in Equation (3), we use the thermal conductivities at the borders between two voxels, where each voxel has six borders and the conductivities on the borders are determined as the harmonic average of the conductivities of the corresponding neighboring voxels for compliance with the law of conservation of energy. For example:

$$
k_{x+0.5, y, z}=2 k_{x, y, z} k_{x+1, y, z} /\left(k_{x, y, z}+k_{x+1, y, z}\right)
$$

At infinity, we assume a constant temperature, and thus, the discretized boundary condition at the edges of the grid is set to $T=25{ }^{\circ} \mathrm{C}$.

For each voxel located at $(x, y, z)$, we can further discretize Equation (1) using the backward Euler method, as follows:

$$
\rho C_{p} \frac{\left(T_{x, y, z ; m+1}-T_{x, y, z ; m}\right)}{d t}=k \cdot\left(\nabla_{r ; m+1}^{2} T\right)+S
$$

where $m$ and $m+1$ represent two successive time points. Reorganization of Equation (5) yields the following equation:

$$
\rho C_{p} T_{x, y, z ; m+1}-d t \cdot k \cdot\left(\nabla_{r ; m+1}^{2} T\right)=\rho C_{p} T_{x, y, z ; m}+d t \cdot S
$$

To solve the linear system of equations derived from Equation (6), we could use either direct or iterative methods. We use the iterative generalized minimum residue method ${ }^{21}$, which saves both computational time and memory in deriving solutions.

\section{Phase model}

As previously assumed in phase models for low concentrations of nanoparticles, we assume straight propagation of light through the sample, with negligible diffraction and refraction effects. Therefore, we assume optical phase changes only, without amplitude changes. In addition, our phase model does not account for the refractive index of the metal itself and instead considers only the refractive index of the medium surrounding the nanoparticles.

The optical phase is proportional to the optical path delay (OPD) of the light interacting with the sample, which is defined as the product of the refractive index differences and the physical path delay of the light ${ }^{22}$. The OPD is accumulative over the entire sample thickness because it accounts for the sum of the differences between the optical route of the light beam that is interacting with the sample and the reference beam that is not subject to the spatial modulation imposed by the sample. At each $(x, y)$ point, the OPD as a function of the temperature $T$ is defined by the following equation:

$$
\mathrm{OPD}(T)=\int L(T) \times n(T) d z
$$

where $n$ is the refractive index of the sample, $L$ is the physical path delay of the light beam, and the integral represents accumulation over the sample thickness. When the temperature changes from $T_{m}$ to $T_{m+1}$, both the refractive index and the physical path delay change. Under the assumption of three-dimensional (3-D) volume expansion caused by such a temperature change, the change in the physical route in the axial direction can be obtained as followed:

$$
L\left(T_{m+1}\right)=L\left(T_{m}\right) \times\left[\rho\left(T_{m}\right) / \rho\left(T_{m+1}\right)\right]^{1 / 3}
$$

where $\rho$ is the solution density.

To assess the change in the optical route caused by a change in the temperature of the surrounding water, we use the dependence of the water density $\rho\left(\right.$ in $\left.\mathrm{kg} \times \mathrm{m}^{-3}\right)$ on the temperature $T(\text { in } \mathrm{K})^{23}$. By fitting a thirddegree polynomial curve to thermodynamic tables listing the density of water at different temperatures with $R^{2}=0.99997$, it is found that one can express the density of water as a function of temperature as follows:

$$
\rho(T)=1.626 \times 10^{-5} T^{3}-1.934 \times 10^{-2} T^{2}+6.95 T+213.8
$$

The relationship among the refractive index of water $n$, the density of water $\rho$, and the temperature was discussed previously ${ }^{24,25}$. This formulation is based on the Lorentz-Lorenz equation, accounting for the molar refraction of water in relation to wavelength, and has been fitted to a selected set of accurately measured refractive-index data points. It can be written as follows:

$$
\begin{gathered}
\frac{n^{2}-1}{n^{2}+2}\left(\frac{1}{\bar{\rho}}\right)=a_{0}+a_{1} \bar{\rho}+a_{2} \bar{T}+a_{3} \bar{\lambda}^{2} \bar{T}+ \\
\frac{a_{4}}{\bar{\lambda}}+\frac{a_{5}}{\bar{\lambda}^{2}-\bar{\lambda}_{U V}^{2}}+\frac{a_{6}}{\bar{\lambda}^{2}-\bar{\lambda}_{I R}^{2}}+a_{7} \bar{\rho}^{2},
\end{gathered}
$$

where $\bar{\rho}=\rho / 1000, \bar{T}=T / 273.15, \bar{\lambda}=\lambda / 589, a_{0}=0.244, a_{1}=9.746 \times 10^{-3}$, $a_{2}=-3.732 \times 10^{-3}, a_{3}=2.687 \times 10^{-4}, a_{4}=1.589 \times 10^{-3}, a_{5}=2.459 \times 10^{-3}$, $a_{6}=0.9, a_{7}=-1.666 \times 10^{-2}, \bar{\lambda}_{U V}=229$, and $\bar{\lambda}_{I R}=5432$.

When working with numerical models rather than analytical solutions, it is possible to use nonlinear relations to describe the relationships between temperature and refractive index and between temperature and material density. These nonlinear relations are highly important in the attempt to understand the dependence of phaserelated signals and temperature. Figure la shows the refractive index as a function of temperature for $\lambda=632.8 \mathrm{~nm}$ (e.g., helium-neon laser probing). The dashed green curve represents the linear relation between refractive index and temperature, as assumed in previous models ${ }^{11,12,14,26}$. The solid blue curve represents the nonlinear relation derived from Equation (10).

We can see that both the linear and nonlinear curves coincide at $25^{\circ} \mathrm{C}$, such that the linear approximation holds within a limited range of temperatures, whereas for large temperature changes of more than 15 degrees (especially useful for PT therapy), the linear relation fails with a large error. Figure $1 \mathrm{~b}$ describes the computed change in the cumulative OPD through a $100 \mu \mathrm{m}$ water slab as a function of the change in temperature (relative to a starting temperature of $25^{\circ} \mathrm{C}$ ) for the linear and nonlinear relations between refractive index and temperature. From these graphs, we can see that for a temperature change of $1^{\circ} \mathrm{C}$, the error in the OPD between the linear and nonlinear curves is $2 \%$, whereas for a change of $40^{\circ} \mathrm{C}$, the error increases to $35 \%$. This nonlinear relation affects the phase measurements and induces increasingly large errors for larger temperature changes if one applies the inaccurate linear relation used in previous works.

\section{Experimental system}

In order to experimentally validate our model, an optical system was built to acquire the PT phase signals of photothermally stimulated nanoparticle solutions. The PT phase signal is defined as the magnitude of OPD oscillations at the temporal frequency of the PT excitation multiplied by $2 \pi$ and divided by the wavelength. 
a
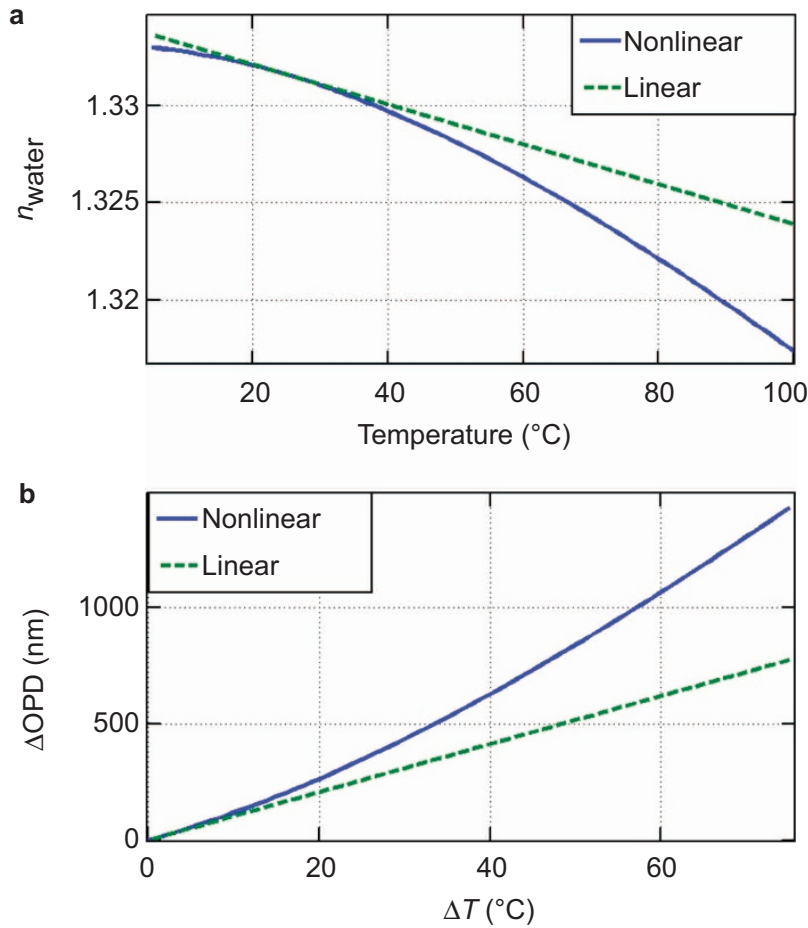

Figure 1 (a) Refractive index of water as a function of temperature according to the linear relation (dashed line) and according to the nonlinear relation (solid line), both at $\lambda=632.8 \mathrm{~nm}$ and starting temperature of $25^{\circ} \mathrm{C}$. (b) The coinciding change in the OPD as a function of the temperature change for a $100 \mu \mathrm{m}$ water slab for the linear relation (dashed line) and for the nonlinear relation (solid line). $\Delta T$ is defined as the instantaneous temperature minus the starting temperature. $\triangle O P D$ is defined as the OPD at the instantaneous temperature minus the OPD at the starting temperature.

Figure 2 depicts the wide-field interferometric phase microscopy system used for the experimental validation of the model. This system is illuminated by two different light sources: a coherent helium-neon $(\mathrm{HeNe})$ laser $(100 \mathrm{~mW}, 632.8 \mathrm{~nm})$ for interferometric imaging and a diode-pumped solid-state (DPSS) laser at a wavelength of $808 \mathrm{~nm}$ for optical excitation of nanorods. The DPSS laser is connected to a waveform generator, which allows for the temporal modulation of the excitation beam. A dichroic mirror (cut-off wavelength of $700 \mathrm{~nm}$ ) is used to combine the two laser beams illuminating the sample. The DPSS laser beam is expanded in 1:6 ratio using two spherical lenses L0 and $\mathrm{L} 1$ (with focal lengths of $2.5 \mathrm{~cm}$ and $15 \mathrm{~cm}$, respectively) and then focused onto the sample using lens L2, with a focal length of $40 \mathrm{~cm}$. The HeNe laser beam passes through the sample, is magnified by a 0.65 NA, $40 \times$ microscope objective (MV-40X, Newport) and projected onto the image plane by spherical tube lens L3 (with a focal length of $16 \mathrm{~cm}$ ). A short-pass filter (cut-off wavelength of $700 \mathrm{~nm}$ ) is positioned after the microscope objective to block the excitation beam from continuing through the imaging channel. The interference is created after the image plane by the external off-axis interferometric module. In this module, after the image is split by a slightly tilted beam splitter, BS, one beam is optically Fourier transformed using lens L4 (with a focal length of $10 \mathrm{~cm}$ ), and in the Fourier plane, a $30 \mu \mathrm{m}$ pinhole is positioned in front of a mirror to back reflect only the dc spatial frequency through lens L4 and the beam splitter onto the camera. This configuration effectively erases the spatial modulation imposed by the sample and allows this beam to function as a reference beam. In the sample arm of the interferometer, lenses L5 and L6 (with focal lengths of $5 \mathrm{~cm}$ each), positioned in a $4 \mathrm{f}$ lens configuration, project the sample image onto a slightly titled mirror, which reflects the beam back to the camera through the same $4 \mathrm{f}$ lens configuration (L5, L6) and the beam splitter. Note that the focal length of lens L4 is twice that of either lens L5 or lens L6 to ensure optical beam-path matching between the interferometric arms. The reference and sample beams interfere on the camera at a small off-axis angle, which creates an off-axis interferogram on the camera; thus allowing for OPD profile reconstruction from a single camera exposure ${ }^{27}$. We used a CCD camera (EPIX 643M) to record interferograms of $160 \times 160$ pixels at $2 \mathrm{kHz}$ for 2 seconds. Digital reconstruction of the sample OPD was performed offline using MATLAB software to spatially filter one of the cross-correlation orders resulting after a digital Fourier transform of the recorded off-axis interferogram ${ }^{28}$. This yielded the complex wave front of the

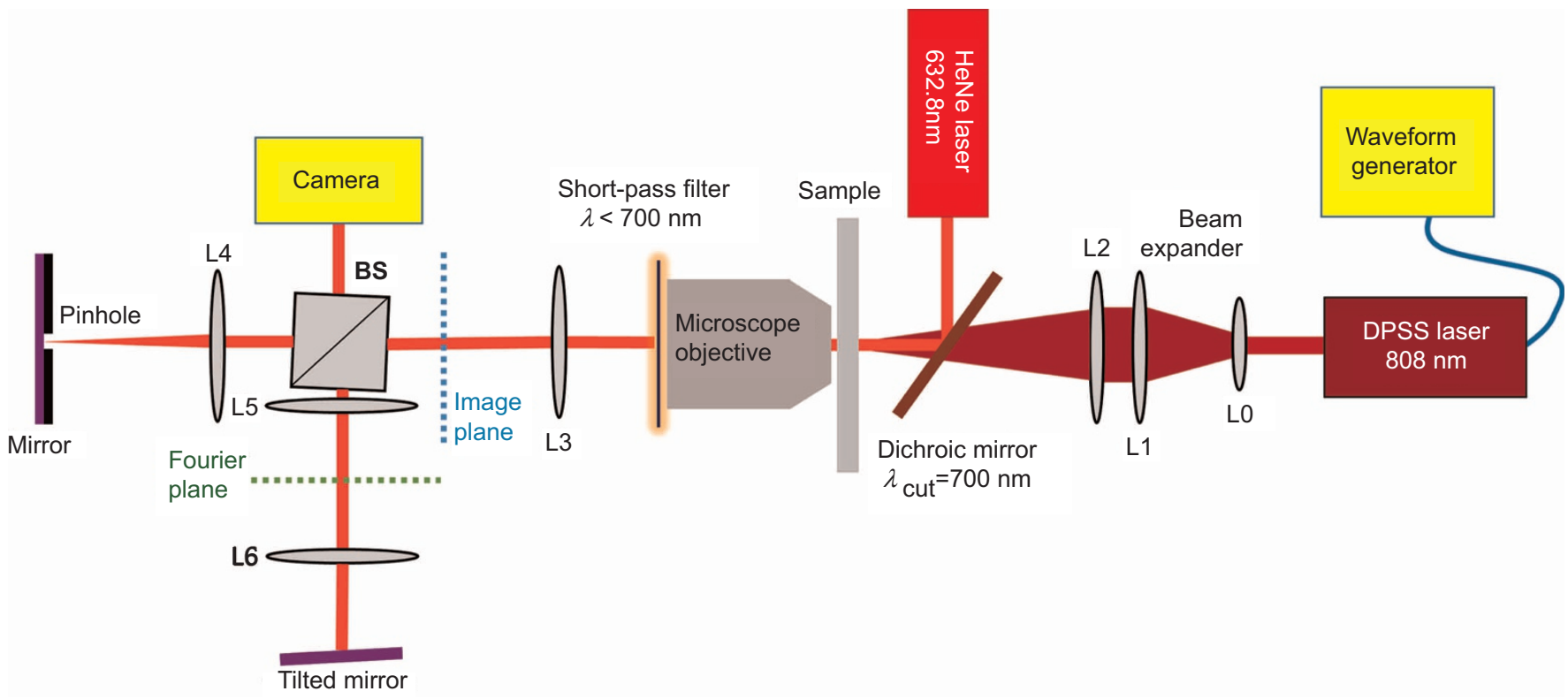

Figure 2 Optical interferometric system scheme used for the experimental measurements. 
light transmitted through the sample. This complex wave front contains the phase profile of the sample, which is proportional to its OPD profile. Since a sequence of interferograms is acquired, a dynamic OPD map is obtained. The PT signal can then be extracted at the temporal frequency of the excitation source ${ }^{15,29}$.

\section{RESULTS AND DISCUSSION}

\section{Model demonstrations for single nanospheres and nanorods}

To simulate the thermal and phase distributions around nanoparticles, we used the heat and phase models described above. To ensure a reasonable computation time, the size of the grid in the heat model was dependent on the period of observation. For 50 ns of observation, the voxel size in the grid was $125 \mathrm{~nm}^{3}$. For $20 \mathrm{~ms}$ of observation, the voxel size in the grid was $1 \mu \mathrm{m}^{3}$. In total, the grid contained 6 million points. To avoid temperature build-up in the sample, the PT phase signal was modulated in time as a result of the time-modulated excitation. Hence, we created a 3-D map of the thermal distribution at each time point and then observed its temporal dependence. Figure 3 illustrates the use of the proposed computational model for nanospheres. In this demonstration, we used a gold nanosphere of $2 R=55 \mathrm{~nm}$ in diameter absorbing $300 \mathrm{fJ}$ over $50 \mathrm{~ns}$.

The time-modulated intensity of the excitation laser is presented in Figure 3a. The resulting 3-D map of the thermal distribution after $5 \mathrm{~ns}$ is presented in Figure 3b. This map shows equal-temperature envelopes around the particle at constant temperatures of 20,10 , and $5{ }^{\circ} \mathrm{C}$ above the starting temperature of $25^{\circ} \mathrm{C}$. For each time point, two-dimensional OPD maps were extracted from the thermal distribution by integrating over the $z$-axis using Equation (7). The resulting two-dimensional map is shown in Figure 3c, where the maximal OPD at a time of $5 \mathrm{~ns}$ is 0.056 $\mathrm{nm}$ (see the entire dynamics in Supplementary Video 1). Figure 3d shows two oscillating OPD signals, the first one at the particle center (solid line) and the second one at a distance $R$ from the particle center (dashed line). Figure $3 e$ shows the Fourier power spectrum of the timemodulated signals presented in Figure $3 \mathrm{~d}$, after subtraction of a quadratic fit from the temporal signals to remove the effect of temperature build-up over time ${ }^{14}$.

Next, we verified the results of our model for a $2 R=50 \mathrm{~nm}$ gold nanosphere stimulated by continuous-wave (CW) laser excitation. The numerical simulation generated a heat distribution map for the particle and its environment during 300 ns of constant excitation, starting at room temperature of $25{ }^{\circ} \mathrm{C}$. Figure 4 shows the dependence of the temperature in time for various ratios of the distance from the nanosphere center $r$ to the nanosphere radius $R$. These results are well consistent with previous finite-element models of heat conduction ${ }^{7}$.

One of the advantages of the presented model compared with previous ones is the possibility of analyzing the thermal and phase signatures of arbitrarily shaped nanoparticles, including semi-symmetric and even non-symmetric shapes, instead of only spheres. Figure 5 shows simulation results of the thermal and OPD distributions produced by using time-modulated excitation of: a nanorod with diameters of $55 \mathrm{~nm}$ $\times 150 \mathrm{~nm}$ (Figure $5 \mathrm{a}$ and Supplementary Video 2 for the full dynamics), a nanocube of $55 \mathrm{~nm} \times 55 \mathrm{~nm} \times 55 \mathrm{~nm}$ in size (Figure $5 \mathrm{~b}$ and Supplementary Video 3 for the full dynamics), and a nanocage of 55 $\mathrm{nm} \times 55 \mathrm{~nm} \times 55 \mathrm{~nm}$ in size, with an edge thickness of $10 \mathrm{~nm}$ (Figure $5 \mathrm{c}$ and Supplementary Video 4 for the full dynamics).

As seen in these figures and videos, surfaces at identical temperatures create different geometrical shapes depending on the distance from the particle surface. Close to the particles (i.e., red envelopes), the equi-temperature surfaces resemble the particle shape, whereas farther from the particles (i.e., blue envelopes), the equi-temperature surfaces resemble a spherical shape. Therefore, the behavior of the associated phase map
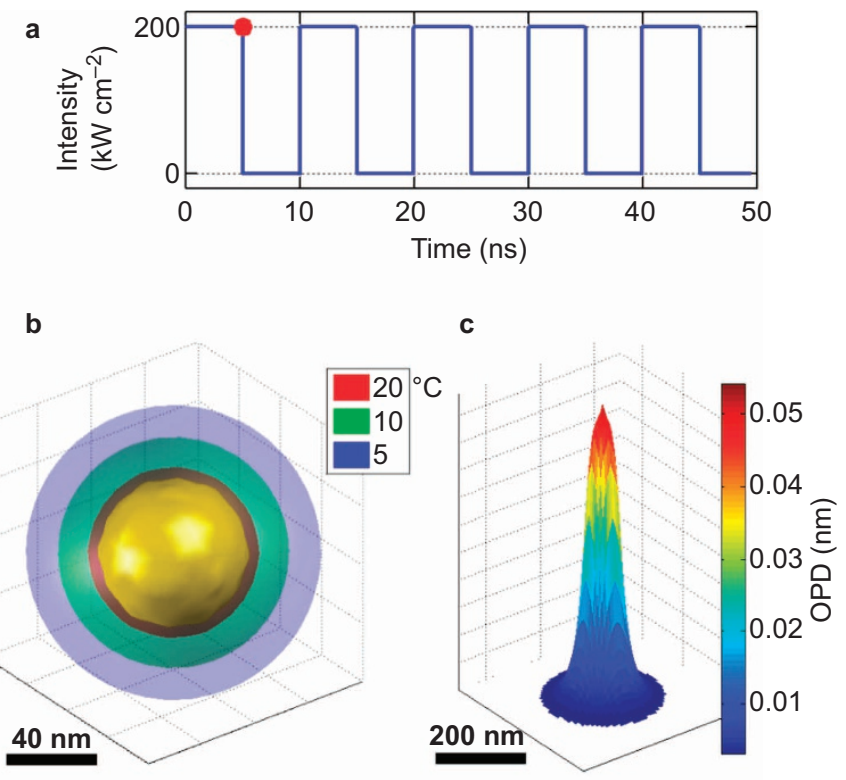

d
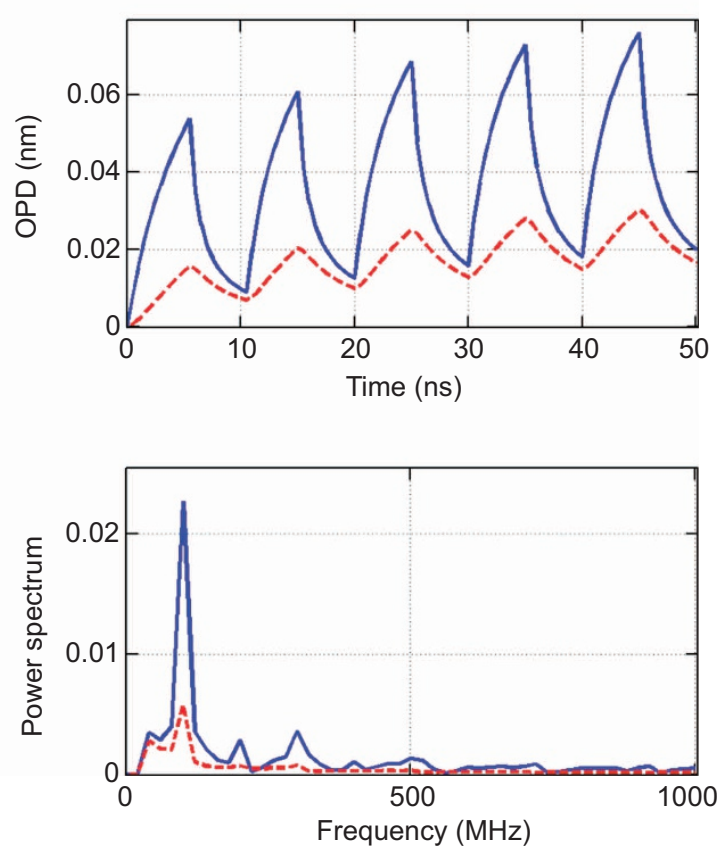

Figure 3 Demonstration of the use of the proposed model for simulating the thermal and OPD distributions of a single nanosphere of $2 R=55 \mathrm{~nm}$ in diameter, absorbing $300 \mathrm{fJ}$ over $50 \mathrm{~ns}$. (a) The intensity of the excitation laser on the sample as a function of time for an excitation frequency of $100 \mathrm{MHz}$. (b) The 3-D map of the thermal distribution $5 \mathrm{~ns}$ after the beginning of the excitation, at the red point marked in a. (c) The corresponding OPD map. See the full dynamics in Supplementary Video 1. (d) The OPD at the central point of the nanosphere (solid-line graph) and at a distance $R$ from the center of the nanoparticle (dashed-line graph) as a function of time. (e) The corresponding power spectra.

measured close to the particle can provide information on the material, shape, and size of the nanoparticle, as previously suggested ${ }^{30}$.

In addition to analysis of arbitrary nanoparticle shapes, the proposed model can also be used to calculate the thermal and OPD distributions around arbitrary number of nanoparticles at arbitrary locations. For example, Figure 6 and Supplementary Videos 5-7 present the 

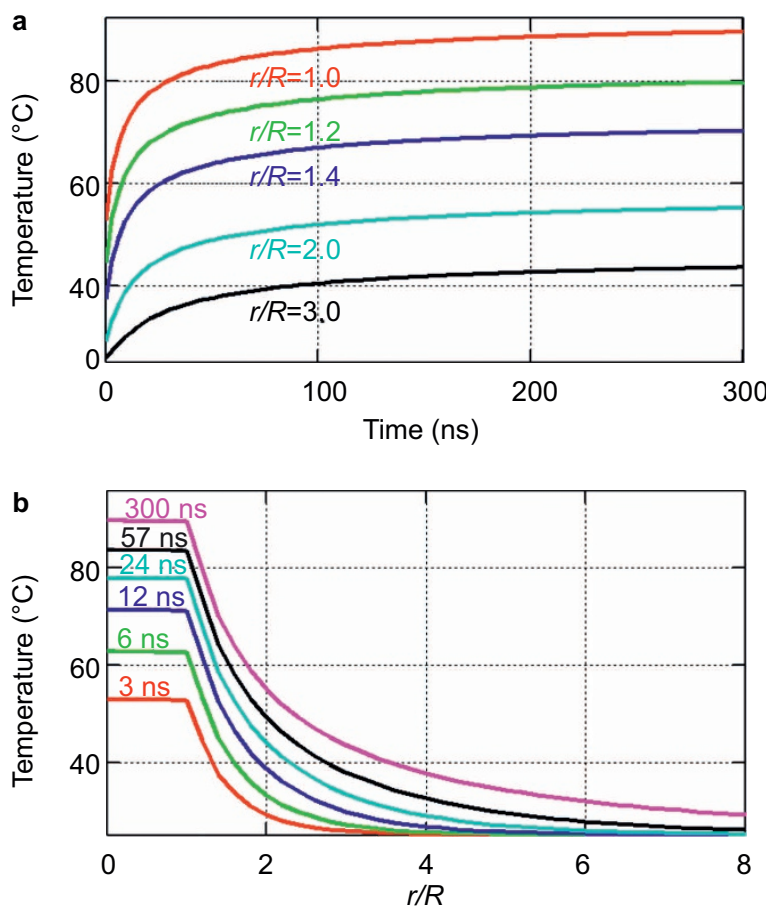

Figure 4 Thermal temporal and spatial dependencies for a $2 R=50 \mathrm{~nm}$ gold nanosphere. (a) Temperature as a function of time for various ratios of the distance from the particle center $r$ to the radius of the particle $R$. (b) Temperature as a function of the ratio of the distance from the particle center $r$ to the radius of the particle $R$ for various time points.

thermal and OPD distributions that result from the time-modulated excitation of two, three, and four gold nanorods. As seen in the two latter cases, a new mutual OPD peak forms over time in the central location among the particles (see the ends of Supplementary Videos 6 and 7) because of temperature build-up.

\section{Experimental verification for nanorod solutions}

The wide-field interferometric phase microscopy system presented in Figure 2 was constructed for measuring the PT phase signals of nanoparticle solutions. The measurements performed were used to verify our computational model.

Simulated and experimental data were collected for $10 \mathrm{~nm} \times 40 \mathrm{~nm}$ gold nanorods at a peak resonance wavelength of $808 \mathrm{~nm}$, an excitation power range of $0-100 \mathrm{~mW}$, an excitation frequency range of $70-1000 \mathrm{~Hz}$, and a solution concentration range of $30-530$ particles/pL. We diluted the nanoparticle solution in deionized water, and then imaged it in 100 $\mu \mathrm{m}$ deep silicon well. PT excitation of the nanoparticle solution created heat absorption, following a phase change, which was not present in the pure water measurements. In the simulations, the nanoparticle excitation power and the laser beam shape were modeled to resemble the experimental optical system setup.

Simulated and experimental results representing the spatially averaged PT phase signal over a field of view of $39.6 \mu \mathrm{m} \times 39.6 \mu \mathrm{m}$ as a function of the excitation laser power are shown in Figure $7 \mathrm{a}$. As expected, an increase in the excitation laser power caused the PT phase signal originating from the nanoparticle solution to intensify. However, in contrast to the results of previous studies ${ }^{14,26}$, we observed a nonlinear relation between the averaged PT phase signal and the excitation power. This can be explained in terms of the nonlinear relation between the phase change and the medium temperature. As seen in Figure 7a, a
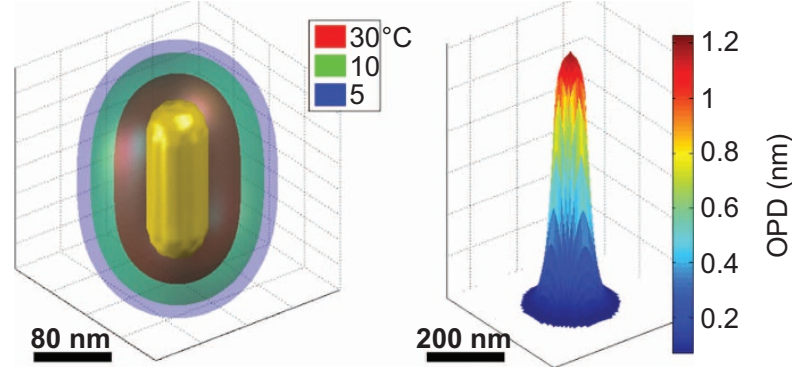

b
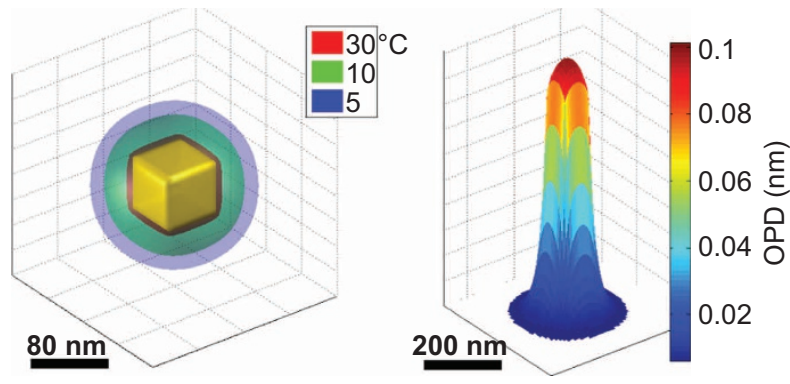

c
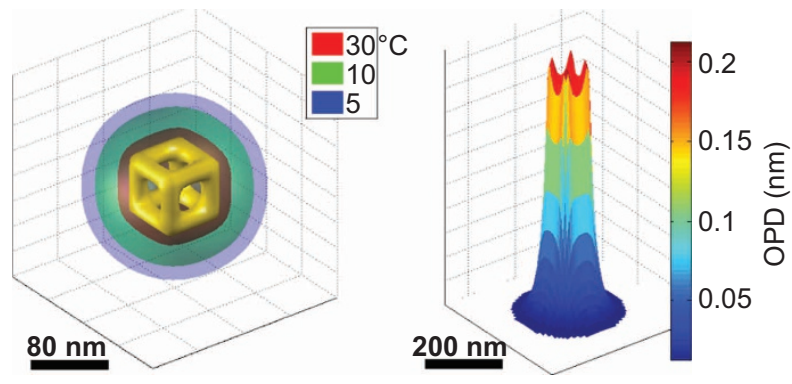

Figure 5 Demonstration of the use of the proposed model to simulate the thermal and OPD distributions of gold nanoparticles of various shapes. (a) Thermal and OPD distributions around a nanorod with diameters of $55 \mathrm{~nm} \times 150 \mathrm{~nm}$, absorbing $260 \mathrm{fJ}, 5 \mathrm{~ns}$ after the beginning of the excitation. See the full dynamics in Supplementary Video 2. (b) Thermal and OPD distributions around a nanocube of $55 \mathrm{~nm} \times 55 \mathrm{~nm} \times 55 \mathrm{~nm}$ in size absorbing $120 \mathrm{fJ}, 5 \mathrm{~ns}$ after the beginning of the excitation. See the full dynamics in Supplementary Video 3. (c) Thermal and OPD distributions around a nanocage of $55 \mathrm{~nm} \times 55 \mathrm{~nm} \times 55 \mathrm{~nm}$ in size with an edge thickness of $10 \mathrm{~nm}$, absorbing $333 \mathrm{fJ}, 5 \mathrm{~ns}$ after the beginning of the excitation. See the full dynamics in Supplementary Video 4.

good agreement was obtained between the simulation results from the proposed model and the experimental results measured using the optical system depicted in Figure 2.

Figure $7 \mathrm{~b}$ presents the simulation and experimental results for the spatially averaged PT phase signal as a function of the excitation laser modulation frequency. The excitation modulation frequency and the pulse duty cycle affect both the maximum temperature of the nanoparticle and the magnitude of the phase oscillations. As seen in Figure $7 \mathrm{~b}$, good agreement was achieved between the simulation results from the model and the experimental measurements obtained by using the optical system depicted in Figure 2. Increasing the excitation frequency shortens the heating-cooling cycle time, the amplitude of the phase signal, and ultimately, the PT phase signal originating from each particle. A change in the heating-cooling cycle time also affects the thermal diffusion radius, such that a higher excitation frequency results in a smaller diffusion radius; thus making the thermal disturbance more confined. This, in turn, causes the phase signal to reduce in diameter, which can be observed using fast imaging 
a

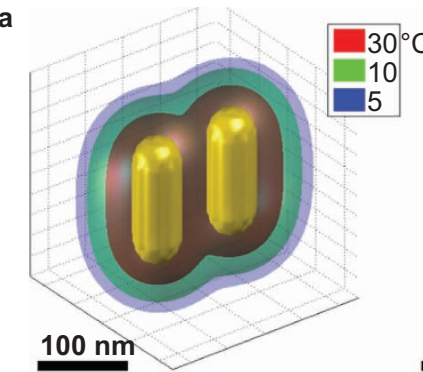

b

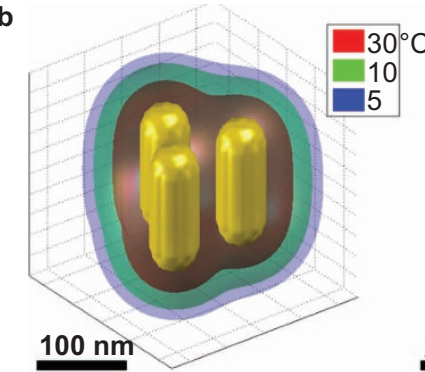

C

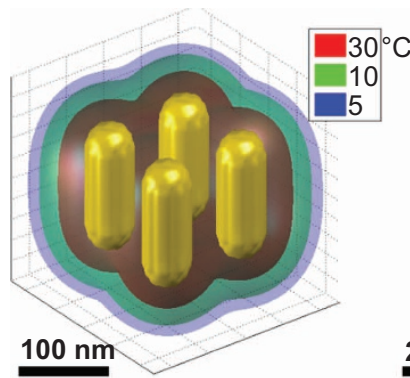

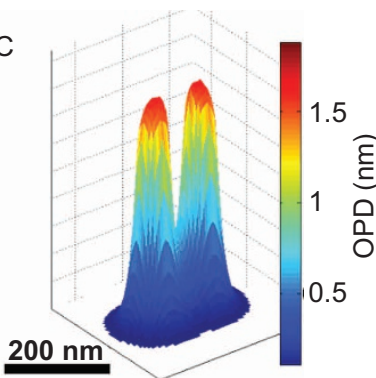

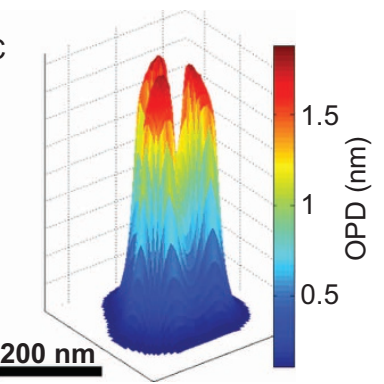

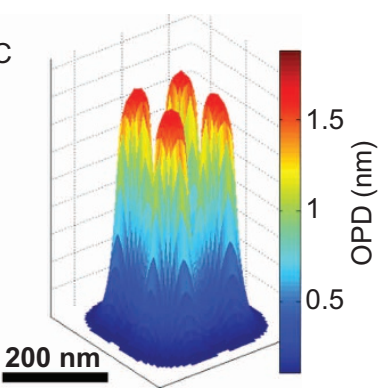

Figure 6 Demonstration of the use of the proposed model to simulate the thermal and phase distributions of groups of multiple particles. We present the thermal and OPD distributions of nanorods, each with dimensions of $55 \mathrm{~nm} \times 150 \mathrm{~nm}$, absorbing $260 \mathrm{fJ}, 5 \mathrm{~ns}$ after the beginning of the excitation.

techniques for detection of phase changes, wherein one can observe the phase distribution and calculate the spread of the thermal disturbance as a function of time ${ }^{31}$. This reduction in the diameter of the PT phase signal allows for a clearer distinction between adjacent particles but at the same time the strength of the PT phase signal decreases.

Figure $7 \mathrm{c}$ presents a comparison between the simulation results, obtained using the proposed model, and the experimental results, obtained using the optical system depicted in Figure 2, concerning the dependence of the spatially averaged PT phase signal on the nanoparticle concentration, again with good agreement between these results. As implied from these graphs, the nanoparticle concentration in the solution affects the optical cross section for energy absorbance and the proximity of individual nanoparticles. A higher concentration of particles induces a stronger PT phase signal in a nonlinear manner. This nonlinearity can be primarily attributed to the superposition of the thermal envelopes of the nanoparticles, which gives rise to a nonlinear effect that grows with particle proximity. The increase in the signal is caused by both the linearly increasing cross section of the nanoparticles and the nonlinear dependency of phase and temperature. This nonlinear effect might be exploited in the future for proximity measurements of particles on the $z$ axis, as when two particles become closer together, the emitted PT phase signal increases.
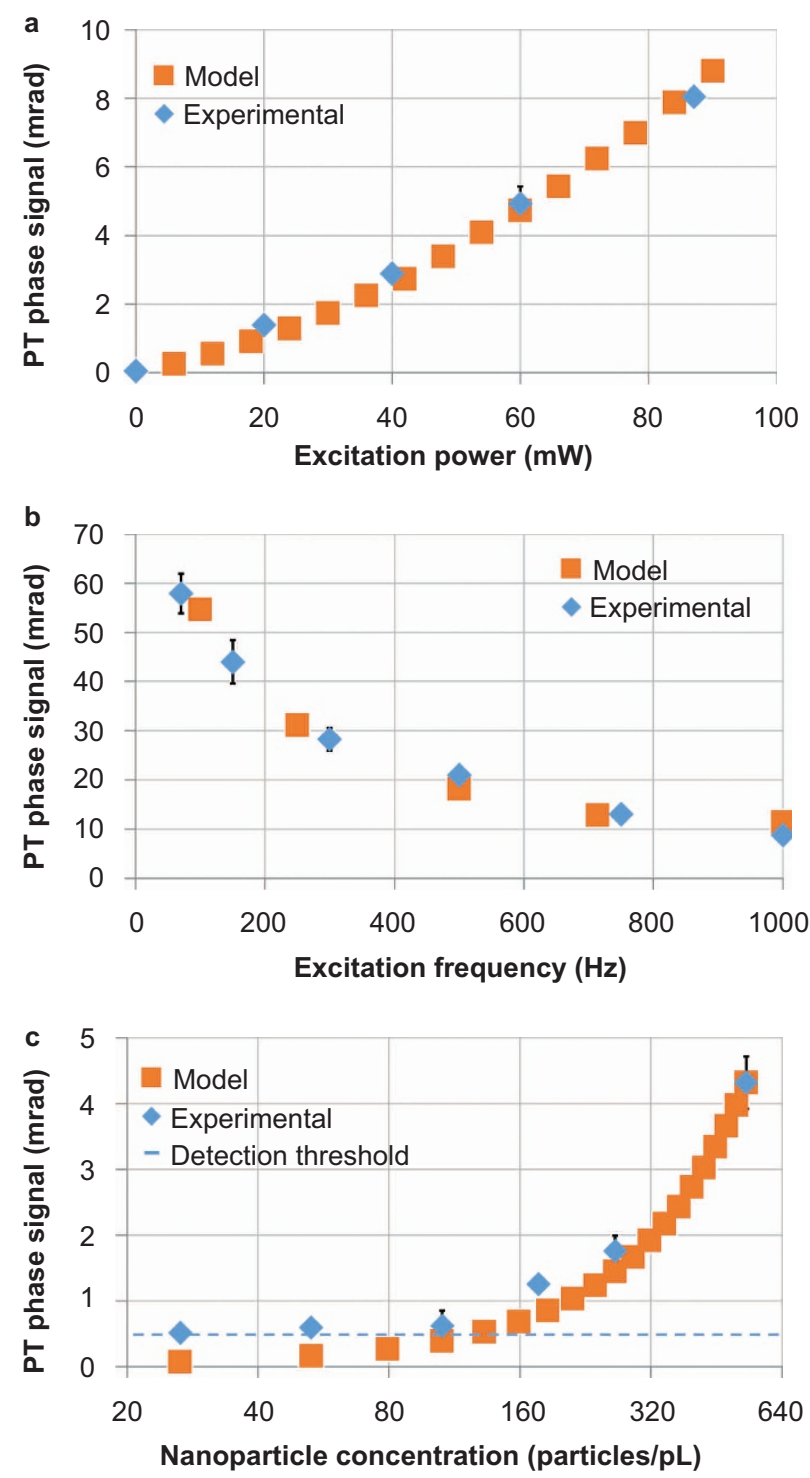

Figure 7 Comparison between the results obtained using the proposed model and the experimental results for a solution of gold nanorods. (a) Spatially averaged PT phase signal as a function of the excitation power $\left(R^{2}=0.991\right.$ correlation between the graphs). (b) Spatially averaged PT phase signal as a function of the excitation laser frequency at duty cycle of $50 \%\left(R^{2}=0.987\right.$ correlation between the graphs $)$. (c) Spatially averaged PT phase signal as a function of the nanoparticle concentration in logarithmic scale ( $R^{2}=0.93$ correlation between the graphs).

The threshold for experimental detection is shown in Figure $7 \mathrm{c}$ as a dashed horizontal line. Most importantly, the proposed model can be used to deduce PT phase signals even for concentrations that are below the detection threshold of the experimental system.

\section{CONCLUSIONS}

We presented a new computational model that can be used to numerically calculate the thermal distribution maps around arbitrarily shaped nanoparticles under PT excitation and find the corresponding phase maps of the light that would be transmitted through the sample. These particles are typically used to produce selective imaging contrast in the sample or for therapy via sample heating. The presented model can be used to optimize the various parameters of the nanoparticles and their excitation prior to the actual experiment. We compared the 
simulation results obtained using our model to both previous thermal distribution studies of plasmonic nanoparticles and new experimental results obtained using wide-field interferometric phase microscopy. Our results revealed good agreement for various parameters, including the nanoparticle size and concentration, the excitation frequency, and the resulting PT phase profiles. These results confirmed that the proposed nonlinear computational model can be used for the optimization of nanoparticles prior to their synthesis and for the investigation of nanoparticle processes and surface interactions based on their phase signatures. Thus, the presented tool can improve the detection efficiency of phase-sensitive techniques that use nanoparticles by optimizing the optical setup parameters, the excitation parameters, and the particle parameters. In addition, by using our model in combination with prior knowledge of the absorbers, one can potentially measure the far-field phase profiles and utilize them to estimate the heat distributions on and around the nanoparticle.

\section{ACKNOWLEDGEMENTS}

This work was supported by the FP7 Marie Curie Career Integration Grant (CIG) No. 303559.

1 Link S, El-Sayed MA. Shape and size dependence of radiative, non-radiative and photothermal properties of gold nanocrystals. Int Rev Phys Chem 2000; 19: 409453.

2 Huang XH, El-Sayed MA. Plasmonic photo-thermal therapy (PPTT). Alexandria J Med 2011; 47: 1-9.

3 Huang $X$, Jain PK, El-Sayed IH, El-Sayed MA. Determination of the minimum temperature required for selective photothermal destruction of cancer cells with the use of immunotargeted gold nanoparticles. Photochem Photobiol 2006; 82: 412-417.

4 Fixler D, Zalevsky Z. In vivo tumor detection using polarization and wavelength reflection characteristics of gold nanorods. Nano Lett 2013; 13: 6292-6296.

5 Richardson HH, Carlson MT, Tandler PJ, Hernandez P, Govorov AO. Experimental and theoretical studies of light-to-heat conversion and collective heating effects in metal nanoparticle solutions. Nano Lett 2009; 9: 1139-1146.

6 Volkov AN, Sevilla C, Zhigilei LV. Numerical modeling of short pulse laser interaction with Au nanoparticle surrounded by water. App/ Surf Science 2007; 253: 63946399.

7 Sassaroli E, Li KC, O'Neill BE. Numerical investigation of heating of a gold nanoparticle and the surrounding microenvironment by nanosecond laser pulses for nanomedicine applications. Phys Med Biol 2009; 54: 5541-5560.

8 Ekici O, Harrison RK, Durr NJ, Eversole DS, Lee M et al. Thermal analysis of gold nanorods heated with femtosecond laser pulses. J Phys D App/ Phys 2008; 41: 185501.

9 Au L, Zheng DS, Zhou F, Li ZY, Li XD et al. A quantitative study on the photothermal effect of immuno gold nanocages targeted to breast cancer cells. ACS Nano 2008; 2: 1645-1652.

10 Huang XH, El-Sayed IH, Qian W, El-Sayed MA. Cancer cell imaging and photothermal therapy in the near-infrared region by using gold nanorods. J Am Chem Soc 2006; 128: 2115-2120.

11 Cognet L, Tardin C, Boyer D, Choquet D, Tamarat P et al. Single metallic nanoparticle imaging for protein detection in cells. Proc Natl Acad Sci 2003; 100: 11350-11355.
12 Boyer D, Tamarat P, Maali A, Lounis B, Orrit M. Photothermal imaging of nanometersized metal particles among scatterers. Science 2002; 297: 1160-1163.

13 Skala MC, Crow MJ, Wax A, Izatt JA. Photothermal optical coherence tomography of epidermal growth factor receptor in live cells using immunotargeted gold nanospheres. Nano Lett 2008; 8: 3461-3467.

14 Adler DC, Huang SW, Huber R, Fujimoto JG. Photothermal detection of gold nanoparticles using phase-sensitive optical coherence tomography. Opt Express 2008; 16: 4376-4393.

15 Turko NA, Peled A, Shaked NT. Wide-Field interferometric phase microscopy with molecular specificity using plasmonic nanoparticles. J Biomed Opt 2013; 18: 111414.

16 Dewhirst MW, Viglianti BL, Lora-Michiels M, Hanson M, Hoopes PJ. Basic principles of thermal dosimetry and thermal thresholds for tissue damage from hyperthermia. Int J Hyperthermia 2003; 19: 267-294.

17 Huang $\mathrm{XH}$, Kang B, Qian W, Mackey MA, Chen PC et al. Comparative study of photothermolysis of cancer cells with nuclear-targeted or cytoplasm-targeted gold nanospheres: continuous wave or pulsed lasers. J Biomed Opt 2010; 15: 058002.

18 Link S, Mohamed MB, El-Sayed MA. Simulation of the optical absorption spectra of gold nanorods as a function of their aspect ratio and the effect of the medium dielectric constant. J Phys Chem B 1999; 103: 3073-3077.

19 Recktenwald GW. Finite-difference approximations to the heat equation. Class Notes 2004. Available at http://www.f.kth.se/jjalap/numme/FDheat.pdf.

20 Hoffman JD, Frankel S. Numerical Methods for Engineers and Scientists. 2nd ed. New York: CRC Press; 2001. pp 270-279.

21 Saad Y, Schultz MH. GMRES: a generalized minimal residual algorithm for solving nonsymmetric linear systems. SIAM J Sci Stat Comput 1986; 7: 856-869.

22 Murphy DB, Davidson MW. Fundamentals of Light Microscopy and Electronic Imaging. Hoboken, NJ: John Wiley \& Sons; 2012.

23 Moran MJ, Shapiro HN, Boettner DD, Bailey MB. Fundamentals of Engineering Thermodynamics. Hoboken, NJ: John Wiley \& Sons; 2010. pp817-818.

24 Schiebener P, Straub J, Sengers JM, Gallagher JS. Refractive index of water and steam as function of wavelength, temperature and density. J Phys Chem Ref Data 1990; 19: 677-717.

25 Harvey AH, Gallagher JS, Sengers JM. Revised formulation for the refractive index of water and steam as a function of wavelength, temperature and density. J Phys Chem Ref Data 1998; 27: 761-774.

26 Tucker-Schwartz JM, Meyer TA, Patil CA, Duvall CL, Skala MC. In vivo photothermal optical coherence tomography of gold nanorod contrast agents. Biomed Opt Express 2012; 3: 2881-2895.

27 Cuche E, Marquet P, Depeursinge C. Simultaneous amplitude-contrast and quantitative phase-contrast microscopy by numerical reconstruction of Fresnel offaxis holograms. Appl Opt 1999; 38: 6994-7001.

28 Girshovitz P, Shaked NT. Fast phase processing in off-axis holography using multiplexing with complex encoding and live-cell fluctuation map calculation in real-time. Opt Express, 2015; 23: 8773-8787.

29 Turko N, Barnea I, Blum O, Korenstein R, Shaked NT. Detection and controlled depletion of cancer cells using photothermal phase microscopy. J Biophoton: doi:10.1002/jbio.201400095.

30 Baffou G, Quidant R, García de Abajo FJ. Nanoscale control of optical heating in complex plasmonic systems. ACS Nano 2010; 4: 709-716.

31 Zharov VP, Lapotko DO. Photothermal imaging of nanoparticles and cells. IEEE J Sel Top Quantum Electron 2005; 11: 733-751.

\footnotetext{
(c) (i) (2) (2) This license allows readers to copy, distribute and transmit the Contribution cc. transform or build upon the Contribution as long as the resulting work is then distributed under this is a similar license. Readers are not permitted to use the Contribution for commercial purposes. Please read the full license for further details at - http://creativecommons.org/

licenses/by-nc-sa/4.0/
} 